\title{
Infantile systemic lupus erythematous presenting as nephrotic syndrome in a 12-month-old boy: a case report
}

\author{
Ece Mekik Akar ${ }^{1 \oplus}$, Zeynep Birsin Özçakar ${ }^{1 \oplus}$, Nilgün Çakar ${ }^{1 \oplus}$, Saba Kiremitçi ${ }^{2 \oplus}$, \\ Eda Didem Kurt Şükür ${ }^{1 \oplus}$, Suat Fitöz ${ }^{3 \odot}$, Fatoş Yalçınkaya ${ }^{1 \oplus}$ \\ Departments of ${ }^{1}$ Pediatric Nephrology, ${ }^{2}$ Pathology and ${ }^{3}$ Pediatric Radiology, Ankara University Faculty of Medicine, Ankara, Turkey.
}

\begin{abstract}
Background. Systemic lupus erythematous (SLE) is extremely rare in infants and has been reported to be a much more severe disease with higher prevalence of critical organ involvement. Herein we present the clinical and laboratory features of infantile SLE (iSLE) with an onset of nephrotic syndrome (NS) during the first year of life.
\end{abstract}

Case. A 12-month-old boy was suffering from generalized edema for two months. He had thrombocytopenia, hemolytic anemia with positive direct and indirect Coombs tests and proteinuria of nephrotic-range. Other laboratory studies revealed slightly decreased C3, low C1q and normal ANA and C4 levels; anti-phospholipid and anti-cardiolipin antibodies were also found to be negative. Renal biopsy revealed Class IV lupus nephritis. The patient also suffered from massive pulmonary thromboembolism. Complete remission was achieved with steroid, cyclophosphamide, mycophenolate mofetil and anticoagulant therapy.

Conclusion. iSLE should be kept in mind especially in infantile NS with multisystem involvement. Renal biopsy is mandatory for early diagnosis. Although the disease was reported to have poor prognosis, complete remission could be achieved with intensive immunosuppressive therapy.

Key words: infantile lupus, systemic lupus erythematous, lupus nephritis, infantile nephrotic syndrome.

\begin{abstract}
Systemic lupus erythematous (SLE) is a multisystemic auto-immune disease characterized by immune dysregulation and formation of auto-antibodies. The disease has childhood onset in $15-20 \%$ of the cases and is uncommon before the age of 5 years. ${ }^{1}$ In infants, SLE is extremely rare and has been reported to be a much more severe disease with higher prevalence of critical organ involvement. Herein we present the clinical and laboratory features of infantile SLE (iSLE) with an onset of nephrotic syndrome (NS) during the first year of life.
\end{abstract}

\footnotetext{
Ece Mekik Akar

emekik@yahoo.com
}

Received 26th January 2020, revised 19th February 2020, 21st March 2020, 20th May 2020,

accepted 12th September 2020.

\section{Case Report}

A12-month-old male patient was admitted to our hospital with a 2-month history of generalized edema. His mother noticed dark urine for one month. Previously he was a healthy child, born at term with birth a weight of 3,450 gr to nonconsanguineous parents and family history did not reveal a significant disease.

On admission, the height and weight of the patient were within normal range. He was pale and blood pressure was measured as 110/70 mmHg. Physical examination revealed anasarca edema including eyelids, face and pretibial regions and ascites (Fig. 1). Laboratory investigations were as follows: BUN $9 \mathrm{mg} / \mathrm{dl}$, creatinine $0.09 \mathrm{mg} / \mathrm{dl}$, creatinine clearance 389 $\mathrm{ml} / \mathrm{min} / 1.73 \mathrm{~m}^{2}$, total protein $2.82 \mathrm{~g} / \mathrm{dl}$, albumin $1.5 \mathrm{~g} / \mathrm{dl}, \mathrm{LDH} 534 \mathrm{U} / \mathrm{L}$, total cholesterol 350 $\mathrm{mg} / \mathrm{dl}$, hemoglobin $6.7 \mathrm{~g} / \mathrm{dl}$, WBC $18,800 / \mathrm{mm}^{3}$, platelet $52,000 / \mathrm{mm}^{3}$ and reticulocyte $6.8 \%$. 


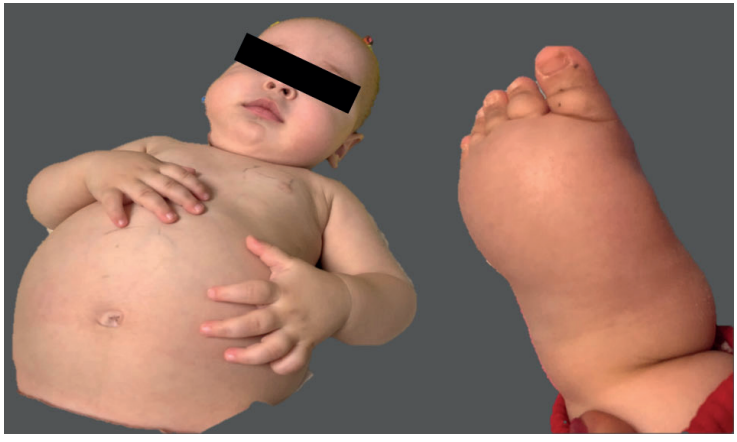

Fig. 1. Pictures of the patient showing anasarca edema and ascites.

Blood smear was consistent with hemolysis. Direct and indirect Coombs tests were found to be positive. Bone marrow aspiration revealed no evidence of malignancy. On the urinalysis, urine density and protein were 1,028 and 1,000 $\mathrm{mg} / \mathrm{dl}$, respectively. Direct microscopy of the urine revealed $545 \mathrm{RBC} / \mathrm{hpf}$ and $83 \mathrm{WBC} / \mathrm{hpf}$. Spot urine protein/creatinine ratio and 24-hour proteinuria were calculated as $21.4 \mathrm{mg} / \mathrm{mg}$ and $496 \mathrm{mg} / \mathrm{m}^{2} /$ hour, respectively. Serum C3 level was $0.73 \mathrm{~g} / \mathrm{L}(\mathrm{N}: 0.86-1.79)$ whereas $\mathrm{C} 4$ level 0.30 $\mathrm{g} / \mathrm{L}(\mathrm{N}: 0.14-0.48)$.

He was diagnosed as NS with generalized edema, nephrotic range proteinuria, hypoalbuminemia and hypercholesterolemia. Renal ultrasonography (USG) showed an increase in both kidney sizes and parenchymal echogenicities.

ANA, anti-dsDNA, lupus anticoagulants, nuclear auto-antibodies and serologic tests for infectious etiologies including EBV, CMV and syphilis were found to be negative. He had a slightly decreased C3 and normal C4 levels, while $\mathrm{C} 1 \mathrm{q}$ levels was found to be low. Antiphospholipid and anti-cardiolipin antibodies were also found to be negative.

Steroid treatment $(8 \mathrm{mg} / \mathrm{kg} / \mathrm{d})$ was commenced for autoimmune hemolytic anemia and thrombocytopenia, and tapered to $2 \mathrm{mg} / \mathrm{kg} / \mathrm{d}$ in 10 days. Echocardiography was normal whereas, thoracoabdominal computed tomography (CT) revealed massive pulmonary thromboembolism (Fig. 2).

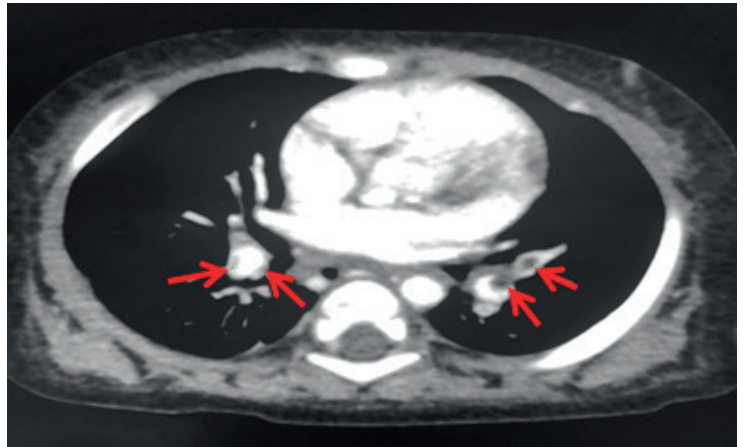

Fig. 2. Pulmonary thromboembolism on computed tomography.

Urgent intravenous (IV) heparin infusion was started. Doppler USG showed additional thrombosis on left deep crural veins. Thrombophilia tests revealed heterozygote mutations for factor $\mathrm{V}$ Leiden and methylenetetrahydrofolate reductase. Anti-thrombotic therapy was continued with enoxaparin. However, respiratory distress started and tachypnea increased soon after. Oliguria developed and hypertension (HT) persisted. His clinic condition was stabilized with repeated blood transfusions, IV albumin infusions and anti-hypertensive medications. Renal biopsy revealed diffuse podocyte hypertrophy, glomerular basal membrane thickening, endocapillary and mesangial proliferation and "full-house" positivity on immunofluorescence staining (including IgG, IgA, C3, C1q, fibrinogen, lambda and kappa) which is consistent with Class IV lupus nephritis (Fig. 3). Pathological vascular findings were not detected on renal biopsy.

In addition to steroid therapy which was slowly tapered after a month of $2 \mathrm{mg} / \mathrm{kg} / \mathrm{d}$ dose, cyclophosphamide (CYP) $\left(500 \mathrm{mg} / \mathrm{m}^{2} /\right.$ dose per month for 6 months) was given. At the third month of therapy, he achieved complete remission with spot urine protein/creatinine ratio $0.3 \mathrm{mg} / \mathrm{mg}$, serum albumin $3.5 \mathrm{mg} / \mathrm{dl}$, and normal renal function tests, hemoglobin, platelet and C3 levels. Mycophenolate mofetil (MMF) $\left(1000 \mathrm{mg} / \mathrm{m}^{2} /\right.$ day) was commenced as maintenance therapy, after 6 monthly doses of CYP were completed. Enoxaparin was given 


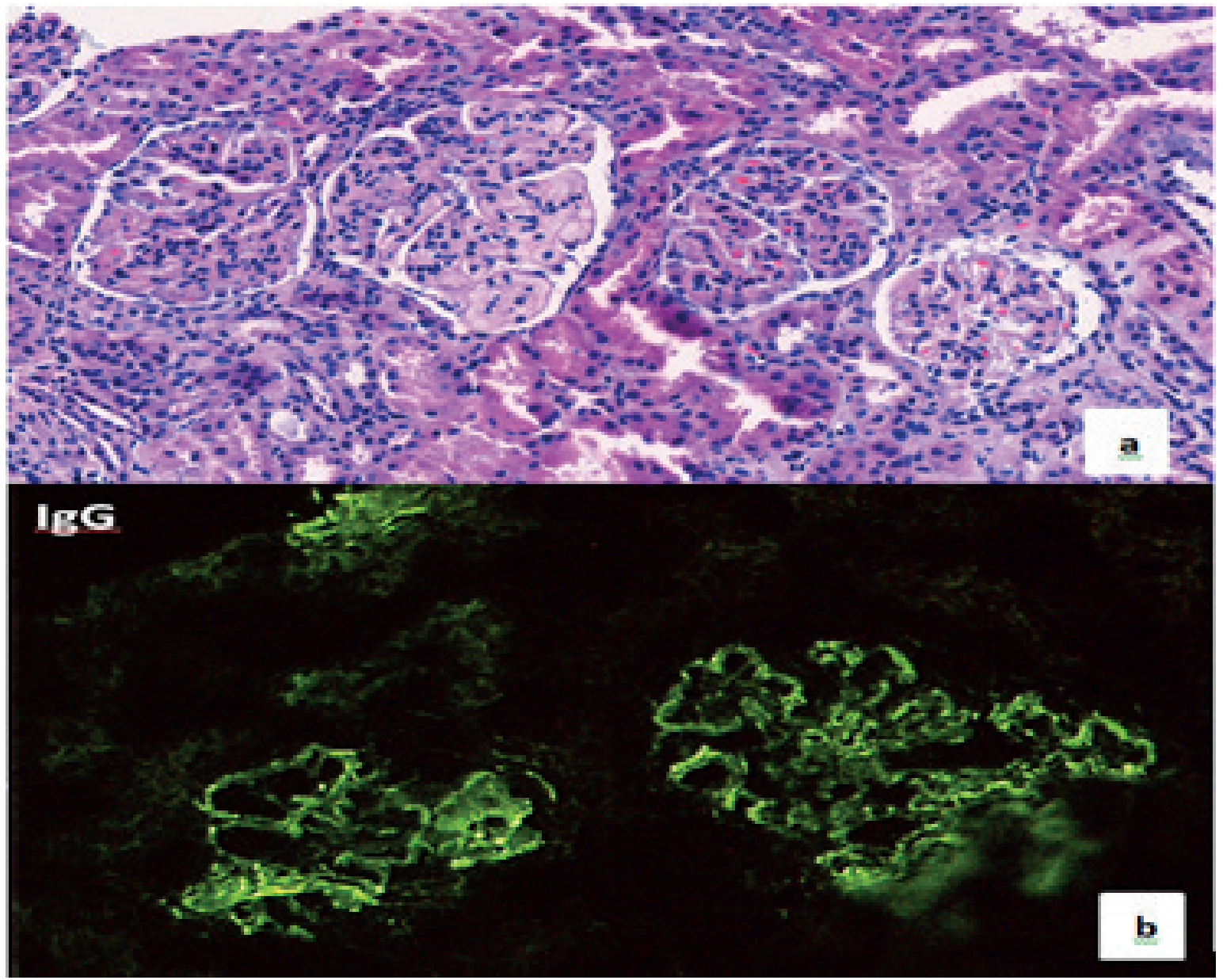

Fig. 3. Renal biopsy specimens: a) endocapillary and mesangial proliferation on light microscopy; b) Ig G depositions on immunofluorescence.

for 6 months and discontinued when control radiologic images (CT and USG) revealed thrombosis-free lungs and extremities.

The last visit of the patient was at the $20^{\text {th }}$ month of the therapy and he is still in complete remission without edema and HT, and with totally normal renal function. Additional genetic work-up for infantile NS (a comprehensive glomerular disease NGS panel) did not reveal any mutations.

Informed consent was obtained from the patient for whom identifying information is included in this article.

\section{Discussion}

Our patient presented with infantile NS developing below 1 year of age which is a rare but severe clinical condition that may occur due to primary or secondary causes. ${ }^{2}$ In regions where consanguineous marriages are prevalent, like our country, primary infantile NS with genetic or syndromic origin is more common. However, he had Coombs positive autoimmune hemolytic anemia and thrombocytopenia that lead us to further investigate for secondary causes. We performed a renal biopsy as soon as possible which revealed lupus nephritis. In spite of his seronegativity for SLE, according 
to SLICC criteria, he was diagnosed as iSLE with his biopsy proven lupus nephritis (LN), autoimmune hemolytic anemia, autoimmune thrombocytopenia and hypocomplementemia. ${ }^{3}$ Though full-house immune staining can also occur in other conditions, he did not have any symptoms suggesting other autoimmune diseases like mixed connective tissue disease (no rash, arthritis or myopathy), Sjögren syndrome (no keratoconjuctivitis sicca), hypocomplementemic urticarial vasculitis syndrome (no typical urticarial rash), IgG4related nephritis, and rheumatoid arthritis (no arthritis).

Infantile lupus with onset before the age of two years is an uncommon disease. It has a more severe disease course as compared with older onset patients. Female predominance that is typical for adult SLE patients is not evident in iSLE with nearly equal incidences in both genders. The interval between the onset of symptoms and diagnosis was found to be shorter and multisystem disease at the time of diagnosis was reported more frequently than older age groups. Noticeably, renal, cardiopulmonary and neurologic involvements have the highest incidence. As a result, iSLE is believed to cause higher morbidity and mortality. ${ }^{1}$ In line with the literature, our patient was male and had two important system involvements at the time of the diagnosis. Additionally, pulmonary thromboembolism which is an important complication of NS aggravated his clinical condition and complicated our treatment.

There are several single case reports about iSLE. $^{4-9}$ Infantile SLE patients presenting with congenital NS as early as 6 weeks of age was reported and all of them had severe progressive multisystemic involvement., ${ }^{4,5}$ Kreindler et al. ${ }^{6}$ presented a 1 month of age male infant with pulmonary hemorrhage and glomerulonephritis. As our patient, he needed an aggressive immunotherapy with a combination of prednisone, intermittent CYP and MMF for a complete serological and clinical remission at 30-month follow-up. The possibility of association between iSLE and Epstein-Barr virus (EBV) infection was suggested in a 14-month-old Japanese boy who had biopsy proven lupus nephritis and hepatitis after an EBV infection.?

To our knowledge, there is only one case series about iSLE reported by Zulian et al. ${ }^{10}$ which included 13 patients with SLE diagnosed in the first year of life. Most common laboratory findings of these patients were anemia, thrombocytopenia and hypocomplementemia which are similar in our patient. Renal disease was the most common organ involvement seen in $92 \%$ of their patients and the most frequent renal pathology was World Health Organization class IV LN. Mortality rate was reported to be high $(38 \%)$ and $62 \%$ of the survivors had residual damage.

Lupus nephritis is believed to be one of the most important predictors of morbidity and mortality in iSLE. Class IV LN, younger age, HT, impaired renal function and low $\mathrm{C} 3$ levels at the time of diagnosis are reported to be indicators of poor prognosis and low treatment response in LN. ${ }^{11}$ While corticosteroids and CYP are the mainstay of induction therapy; azathioprine, MMF, rituximab and tacrolimus are other options which are shown to be efficient. ${ }^{12}$ Following the induction phase, all patients with LN should be administered maintenance therapy to sustain remission. Azathioprine and MMF were shown to be superior to CYP as a maintenance drug, with less serious side effects. ${ }^{11-13}$ Regarding general approach to treatment of infantile LN, our patient was treated with CYP and high dose steroids in induction phase followed by MMF and low dose steroids as maintenance therapy. Despite having severe clinical and laboratory features at presentation, he achieved remission in the third month of therapy and still in remission with good growth.

Although infantile NS usually develops secondary to genetic and syndromic disorders, rare conditions such as iSLE might be the underlying etiology. The possibility of iSLE should be kept in mind especially in infantile NS patients who have multisystemic involvement. 
Renal biopsy is mandatory for early diagnosis. Although the disease was reported to have poor prognosis, complete remission was achieved with intensive therapy. Careful long-term follow up is necessary for early diagnosis of recurrences.

\section{REFERENCES}

1. Pluchinotta FR, Schiavo B, Vittadello F, Martini G, Perilongo G, Zulian F. Distinctive clinical features of pediatric systemic lupus erythematosus in three different age classes. Lupus 2007; 16: 550-555.

2. Holmberg C. JH. Nephrotic syndrome in the first year of life. In: Kher KK, Schnaper HW, Greenbaum LA (eds). Clinical Pediatric Nephrology. London: Taylor and Francis Medical Publishers, 2017: 353365.

3. Petri M, Orbai AM, Alarcon GS, et al. Derivation and validation of the Systemic Lupus International Collaborating Clinics classification criteria for systemic lupus erythematosus. Arthritis Rheum 2012; 64: 2677-2686.

4. Dudley J, Fenton T, Unsworth J, Chambers $\mathrm{T}$, MacIver A,Tizard J. Systemic lupus erythematosus presenting as congenital nephrotic syndrome. Pediatr Nephrol 1996; 10: 752-755.

5. Massengill SF, Richard GA,Donnelly WH. Infantile systemic lupus erythematosus with onset simulating congenital nephrotic syndrome. J Pediatr 1994; 124: 27-31.
6. Kreindler J, Ellis D, Vats A, Kurland G, Ranganathan S,Moritz ML. Infantile systemic lupus erythematosus presenting with pulmonary hemorrhage. Pediatr Nephrol 2005; 20: 522-525.

7. Kishi N, Suga K, Matsuura S, et al. A case of infantile systemic lupus erythematosus with severe lupus nephritis and EBV infection. CEN Case Rep 2013; 2: 190-193.

8. Jordan SC, Lemire JM, Border W, Sakai R, Ettenger RB,Fine RN. False-negative anti-DNA antibody activity in infantile systemic lupus erythematosus (SLE). J Clin Immunol 1984; 4: 156-162.

9. Garg T, Sanke S, Chander R, Agarwal S, Ahmed $R$. Infantile systemic lupus erythematosus with cerebral calcification and delayed development. Indian J Pediatr 2018; 85: 394-395.

10. Zulian F, Pluchinotta F, Martini G, Da Dalt L, Zacchello G. Severe clinical course of systemic lupus erythematosus in the first year of life. Lupus 2008; 17: 780-786.

11. Thakur N, Rai N,Batra P. Pediatric lupus nephritisreview of literature. Curr Rheumatol Rev 2017; 13: 29-36.

12. Contreras G, Pardo V, Leclercq B, et al. Sequential therapies for proliferative lupus nephritis. N Engl J Med 2004; 350: 971-980.

13. Tanaka H, Joh K, Imaizumi T. Treatment of pediatriconset lupus nephritis: a proposal of optimal therapy. Clin Exp Nephrol 2017; 21: 755-763. 